\title{
CARACTERIZACIÓN FENOTÍPICA DEL GÉNERO POLYLEPIS EN EL BOSQUE NATIVO DE LA PROVINCIA DE CANDARAVE-TACNA
}

\author{
PHENOTYPIC CHARACTERIZATION OF GENUS POLYLEPIS IN THE \\ NATIVE FOREST OF THE PROVINCE OF CANDARAVE-TACNA
}

\author{
Luis Morales Aranibar ${ }^{1}$; Giovanni Aragón Alvarado; Edith Delmira Silva Flores ${ }^{3}$
}

\begin{abstract}
RESUMEN
El estudio se realizó en el bosque de Queñoales, cerca al volcánYucamani, entre las coordenadas $70^{\circ} 12^{\prime} 15^{\prime \prime}$ de longitud oeste, $17^{\circ} 15^{\prime} 30^{\prime \prime}$ latitud sur, a una altitud de 3,515msnm., en la Provincia de Candarave, Región Tacna. El propósito fue caracterizar fenotípicamente la vegetación.

Entre las características se determinó la altura promedio $3,52 \mathrm{~m}$, el largo del foliolo $1,51 \mathrm{~cm}$, ancho del foliolo de $0,76 \mathrm{~cm}$, inflorescencia $3,34 \mathrm{~cm}$, flor $0,59 \mathrm{~cm}$, vaina estipular $0,42 \mathrm{~cm}$., tallos torcidos, corteza delgada y exfoliante, de color marrón rojizo. Los foliolos trifoliados; pelos glandulares blancos, raquis lanoso, las vainas estipulares con ápice protuberante y densamente lanoso. Flor de color rojo cereza, dialipétala. Los frutos de color naranja-marrón, con espinas de color negro cubiertos de pelos blancos, caracterización que correspondió a Polylepis rugulosa y no Polylepis besseri como la literatura señalaba.
\end{abstract}

Palabras clave:Polylepis rugulosa, Polylepis besseri, Caracterización Fenotípica, Queñua.

\section{ABSTRACT}

\begin{abstract}
The study was carried out in the queñuales forest, near theYucamani volcano, at an altitude of $3515 \mathrm{msnm}$ in the. Among the coordinates $70^{\circ} 12^{\prime} 15^{\prime} \mathrm{W}, 17^{\circ} 15^{\prime} 30^{\prime \prime} \mathrm{S}$, at a height of 3,515 msnm., Province of Candarave, Tacna Region. The purpose of this study was to phenotypically characterize the Polylepis forest.

Among the features are determined average height $3.52 \mathrm{~m}$, length of $1.51 \mathrm{~cm}$ leaflet, leaflet width of $0.76 \mathrm{~cm}, 3.34 \mathrm{~cm}$ inflorescence, flower $0.59 \mathrm{~cm}, 0.42 \mathrm{~cm}$ sheath stipulate, twisted stems, thin and exfoliating bark, reddish brown. The trifoliate leaflets, glandular hairs white, woolly rachis, stipular pods with bulging apex, densely woolly. Cherry red flower, dialipétala.The fruits orange-brown with black colored spines covered with white hairs, characterization accounted Polylepis Polylepis besseri rugulosa and literature as noted.
\end{abstract}

Key words: Polylepis rugulosa, Polylepis besseri, Phenotypic Characterization, Queñua.

\section{INTRODUCCIÓN}

\section{Los bosques de Queñoales}

Las poblaciones de queñoas están confinadas a los andes tropicales y subtropicales sudamericanos. No existen mapas fitogeográficos detallados en Sudamérica.

En la mayoría de los casos, la ecología de los bosques nativos del Perú es desconocida, debido a la falta de estudios que determinan la verdadera situación de esta vegetación. La presente investigación estudia el bosque de Queñoales que se encuentra a $3515 \mathrm{msnm}$, en la Provincia de Candarave, Región Tacna.

\section{OBJETIVOS}

\section{Objetivo general}

Caracterizar fenotípicamente el Género Polylepis en el bosque nativo de la Provincia de Candarave-Tacna.

\section{Objetivos específicos}

Establecer las características morfológicas externas del género Polylepis en el bosque; así como determinar sus características florales, precisar sus características frutales y realizar un mapa fitogeagráfico.

\footnotetext{
Biólogo Microbiólogo. Facultad de Ciencias de la UNJBG.

${ }^{2}$ Biólogo, Doctor en Ciencias y Tecnologías Ambientales. Facultad de Ciencias de la UNJBG.

${ }^{3}$ Ingeniera Pesquera. Facultad de Ciencias Agropecuarias de la UNJBG.
} 


\section{MÉTODO DE MUESTREO}

Se plantearon 4 transeptos lineales de modo que 1 Transepto $=15$ transeptos pequeños o a partir de un punto de inicio se proyectó una línea o transepto, con una orientación fija, a lo largo del cual se levantó la información sobre la características de la Queñoa.

Se tomaron los siguientes indicadores, descriptores morfológicos fenotípicos: dimensión del foliolo, forma del foliolo, tipo del foliolo, raquis, vainas estipulares, pelos glandulares, glabra, color del foliolo, longitud de la inflorescencia, tamaño de la flor, color de la flor, pelusa de la flor, altura del árbol, forma del fruto, tipo de fruto.

\section{Técnicas de recolección de datos:}

El trabajo de campo: Por cada transepto se eligió un árbol específico y se codificó con GP, se marcó con pintura a fin de reconocerlo, luego se procedió a la colecta. Se tomaron tres muestras de ramas fértiles en floración, luego se colocó en una bolsa etiquetada y en una ficha de antecedentes se registró a las características fenotípicas de cada muestra. El largo y ancho del foliolo se midieron con vernier. La altura del árbol se midió con una cinta métrica de metal. El tamaño de la flor con el vernier. La flor se colectó en un frasco donde se hizo una cama de algodón para que llegue en perfectas condiciones al laboratorio. El fruto se buscó en la flor; ya que es difícil de observar a simple vista, luego, se continúo en el laboratorio con ayuda del estetoscopio.
Trabajo de gabinete: Se hizo el prensado de las muestras. El secado se realizó en medio ambiente por un lapso de 7 días. Luego de procedió a montarlo y etiquetarlo.

\section{Procesamiento y análisis de datos:}

El análisis de información se efectuó a partir de los datos obtenidos de las mediciones. Se elaboró una base de datos, en seguida, se hizo el control de calidad, hasta, finalmente, procesar la información. El análisis de datos se realizó con la aplicación del programa Stata v. 9.0 para Windows (StataCorp LP, Collage Station, Texas, United Status of America). Mediante un análisis descriptivo se calcularon las medidas de tendencia central (media aritmética y desviación Estándar).

Para contrastar y verificar las hipótesis estadística, con relación a la comparación de las mediciones de la altura del árbol, largo de foliolo, ancho de foliolo, inflorescencia, tamaño de flor y vaina estipular, se usó el modelo de análisis de varianza de ANOVA, considerándose, para todos los casos, una confiabilidad de $99.9 \%(\mathrm{p}<0.01)$.

En la presentación de resultados, se utilizó cuadros y gráficos de Caja y líneas, gráficos de intervalo de confianza a fin de poner en evidencia la respuesta al problema.

\section{RESULTADOS Y DISCUSIÓN}

\section{Características fenotípicas cuantitativas:}

Tabla $\mathrm{N}^{\circ}$ 01: Descripción de las características fenotípicas por trasepto de Polylepis rugulosa (QUEÑOA) Provincina de Candarave. 2008-2009.

\begin{tabular}{|c|c|c|c|c|c|c|c|}
\hline Transepto & $\begin{array}{c}\text { Caracteristica } \\
\text { fenotipica }\end{array}$ & $\begin{array}{c}\mathrm{N}^{\circ} \text { de } \\
\text { mediciones } \\
\text { (meses) }\end{array}$ & Mnimo & Maximo & Media & $\mathrm{DE}^{*}$ & cV \\
\hline \multirow{6}{*}{$\begin{array}{c}T 1 \\
(n=15)\end{array}$} & Altura del árbol & 7 & 2,4 & 4,6 & 3,38 & 0,66 & 0,06 \\
\hline & Largo de folido & 7 & 1.3 & 2.0 & 1.69 & 0.18 & 0,02 \\
\hline & Ancho de foliolo & 7 & 0,5 & 2,0 & 0,77 & 0,18 & 0,02 \\
\hline & Inflorescencia & 7 & 2.9 & 3,9 & 3,34 & 0,31 & 0,04 \\
\hline & Tamaño de Flor & 7 & 0.5 & 0.7 & 0.60 & 0,07 & 0.01 \\
\hline & Vaina estipular & 7 & 0.3 & 0.6 & 0.43 & 0,08 & 0.00 \\
\hline \multirow{6}{*}{$\begin{array}{c}T 2 \\
(n=15)\end{array}$} & Altura del árbol & 7 & 1,5 & 5,2 & 3,46 & 1,11 & 0,01 \\
\hline & Largo de foliolo & 7 & 1.0 & 2,0 & 1,42 & 0.22 & 0,01 \\
\hline & Ancho de foliolo & 7 & 0.5 & 1,3 & 0,75 & 0,15 & 0,01 \\
\hline & Inflorescencia & 7 & 2,8 & 3,9 & 3,32 & 0,28 & 0,03 \\
\hline & Tarnaño de Flor & 7 & 0.5 & 0.7 & 0.61 & 0,08 & 0,01 \\
\hline & Vaina estipular & 7 & 0.3 & 0.6 & 0,40 & 0,08 & 0,00 \\
\hline \multirow{6}{*}{$\begin{array}{c}T 3 \\
(n=15)\end{array}$} & Altura del árbol & 7 & 2,4 & 4,9 & 3,66 & 0,80 & 0,07 \\
\hline & Largo de foliolo & 7 & 1.0 & 1,9 & 1,52 & 0,19 & 0,01 \\
\hline & Ancho de foliolo & 7 & 0.4 & 1,2 & 0,78 & 0,17 & 0,01 \\
\hline & Inflorescencia & 7 & 2,9 & 3,9 & 3,37 & 0,27 & 0,03 \\
\hline & Tamaño de For & 7 & 0.5 & 07 & 0.58 & 0.08 & 0.00 \\
\hline & Vaina estipular & 7 & 0.3 & 0.6 & 0.42 & 0,08 & 0,00 \\
\hline \multirow{6}{*}{$\underset{(n=15)}{T 4}$} & Altura del árbol & 7 & 2,8 & 4,7 & 3,61 & 0.70 & 0,06 \\
\hline & Largo de foliolo & 7 & 1,1 & 1,9 & 1,40 & 0,12 & 0,01 \\
\hline & Ancho de foliolo & 7 & 0.5 & 1.0 & 0,74 & 0.13 & 0,01 \\
\hline & Inflorescencia & 7 & 2.8 & 3.8 & 3.34 & 0.29 & 0,03 \\
\hline & Tamaño de Flor & 7 & 0.5 & 07 & 0,59 & 0.07 & 0.00 \\
\hline & Vaina estipular & 7 & 0.3 & 0.6 & 0.43 & 0.08 & 0,00 \\
\hline
\end{tabular}

Fuente: Ficha de recolección de datos.

*Desviación estándar (DE).

* Coeficiente de variabilidad (CV). 
Tabla $\mathrm{N}^{\circ}$ 02: Promedio global de las características fenotípicas de Polylepis rugulosa (Queñoa) provincia de Candarave, 2008-2009.

\begin{tabular}{|c|c|c|c|c|c|}
\hline $\begin{array}{c}\text { Caracteristica } \\
\text { tenotipica }\end{array}$ & $\begin{array}{c}N^{\bullet} \text { de } \\
\text { mediciones } \\
\text { (meses) }\end{array}$ & Minimo & Máximo & Media & DE: \\
\hline $\begin{array}{l}\text { Altura del árbol } \\
(n=60)\end{array}$ & 7 & 1,5 & 5,2 & 3,53 & 0,84 \\
\hline $\begin{array}{l}\text { Largo de foliolo } \\
(n=60)\end{array}$ & 7 & 1,0 & 2,0 & 1,51 & 0,22 \\
\hline $\begin{array}{l}\text { Ancho del to tiolo } \\
(n=60)\end{array}$ & 7 & 0,4 & 2,0 & 0.76 & 0,16 \\
\hline $\begin{array}{l}\text { In florescencia } \\
(n=60)\end{array}$ & 7 & 2,8 & 3,9 & 3,34 & 0.29 \\
\hline $\begin{array}{l}\text { Tamaño de Flor } \\
(n=60)\end{array}$ & 7 & 0,5 & 0,7 & 0,59 & 0,08 \\
\hline $\begin{array}{l}\text { Vaina estipular } \\
(n=60)\end{array}$ & 7 & 0,3 & 0,6 & 0,42 & 0,08 \\
\hline
\end{tabular}

Fuente: Ficha de recolección de datos.

*Desviación Estándar(DE).

Tabla No $\mathbf{N}^{\mathbf{0}}$ : Análisis de varianza (Anova) de la altura del árbol por transecto de Polylepis Rugulosa (Queñoa) provincia Candarave, 2008-2009.

\begin{tabular}{|rccccc|}
\hline & $\begin{array}{c}\text { Suma de } \\
\text { cuadrados }\end{array}$ & g & $\begin{array}{c}\text { Media } \\
\text { cuadratica }\end{array}$ & F & Sig. \\
\hline Afura del artol inter-grupos & 5.328 & 3 & 1,78 & 2,54 & 0.056 \\
Intra-grupos & 290.654 & 416 & 0,70 & & \\
Total & 295.982 & 419 & & & \\
\hline
\end{tabular}

Fuente: Ficha de recolección de datos.

Tabla $N^{\circ}$ 04: Análisis de varianza (ANOVA) del largo del foliolo por transecto de Polylepis rugulosa (Queñoa) provincia Candarave, 2008-2009.

\begin{tabular}{|rrrrrr|}
\hline $\begin{array}{r}\text { Suma de } \\
\text { cuadrados }\end{array}$ & gl $\begin{array}{r}\text { Media } \\
\text { cuadrática }\end{array}$ & F & Sig. \\
\hline Largo del foliolo Inter-grupos & 5,496 & 3 & 1,83 & 54,93 & 0,000 \\
Intra-grupos & 13,874 & 416 & 0,03 & & \\
Total & 19,369 & 419 & & & \\
\hline
\end{tabular}

Fuente: Ficha de recolección de datos.

Tabla N $\mathbf{N}^{\circ}$ 05: Análisis de varianza (ANOVA) del ancho del foliolo por transecto de Polylepis Rugulosa (Queñoa) provincia Candarave, 20082009.

\begin{tabular}{|c|c|c|c|c|c|c|}
\hline & & $\begin{array}{r}\text { Suma de } \\
\text { cuadrados }\end{array}$ & gl & $\begin{array}{r}\text { Media } \\
\text { cuadratica }\end{array}$ & $\mathrm{F}$ & Sig. \\
\hline \multirow[t]{3}{*}{ Ancho del foliolo } & Inter-grupos & 0.095 & 3 & 0.03 & 1,25 & 0.288 \\
\hline & Intra-grupos & 10,489 & 416 & 0,03 & & \\
\hline & Total & 10,584 & 419 & & & \\
\hline
\end{tabular}

Fuente: Ficha de recolección de datos.

Tabla N ${ }^{\circ}$ 06: Análisis de varianza (ANOVA) de la longitud de la inflorescencia por transecto de Polylepis Rugulosa (Queñoa) provincia Candarave, 2008-2009.

\begin{tabular}{|rrrrrrr|}
\hline & & $\begin{array}{r}\text { Suma de } \\
\text { cuadrados }\end{array}$ & gl & $\begin{array}{r}\text { Media } \\
\text { cuadratica }\end{array}$ & F & Sig. \\
\hline Inflorescencia & Inter-grupos & 0,357 & 3 & 0,12 & 0,04 & 0,999 \\
& Intra-grupos & 1187,033 & 416 & 2,85 & & \\
& Total & 1187,390 & 419 & & & \\
\hline
\end{tabular}

Fuente: Ficha de recolección de datos.
Tabla $\mathbf{N}^{\circ}$ 07: Análisis de varianza (ANOVA) del tamaño de la flor por transecto de Polylepis Rugulosa (Queñoa) en la provincia de Candarave Tacna, 2008-2009.

\begin{tabular}{|cccccc|}
\hline & $\begin{array}{c}\text { Suma de } \\
\text { cuadrados }\end{array}$ & gl & $\begin{array}{c}\text { Media } \\
\text { cuadrática }\end{array}$ & F & Sig. \\
\hline Tamaño de Flor Inter-grupos & 0024 & 3 & 0008 & 0008 & 0967 \\
Intra-grup os & 38216 & 415 & 0092 & & \\
Total & 38.240 & 418 & & & \\
\hline
\end{tabular}

Fuente: Ficha de recolección de datos

Tabla $N^{\circ}$ 08: Análisis de varianza (ANOVA) del tamaño de la vaina estipular por transecto de Polylepis Rugulosa (Queñoa) provincia Candarave, 2008-2009.

\begin{tabular}{|c|c|c|c|c|c|c|}
\hline & & $\begin{array}{l}\text { Sumb de } \\
\text { cuadrados }\end{array}$ & gl & $\begin{array}{l}\text { Media } \\
\text { cuadrática }\end{array}$ & $F$ & Sig \\
\hline \multirow[t]{3}{*}{ Vaina estipular } & Inter-grupos & 0,060 & 3 & 0,02 & 3,12 & 0,026 \\
\hline & Intra-grupos & 2,650 & 416 & 0.01 & & \\
\hline & Total & 2,710 & 419 & & & \\
\hline
\end{tabular}

Fuente: Ficha de recolección de datos.

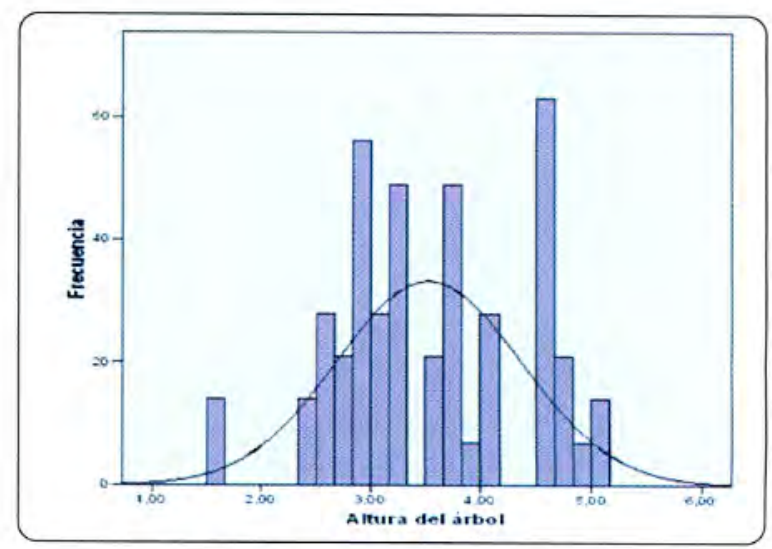

Gráfico $\mathbf{N}^{\mathbf{0}}$ 01: Curva de distribución normal de la altura árbol de polylepis rugulosa por transepto provincia Candarave 2008-2009. Donde: Media - 3.53, desviación típica. 0.84 y N-420

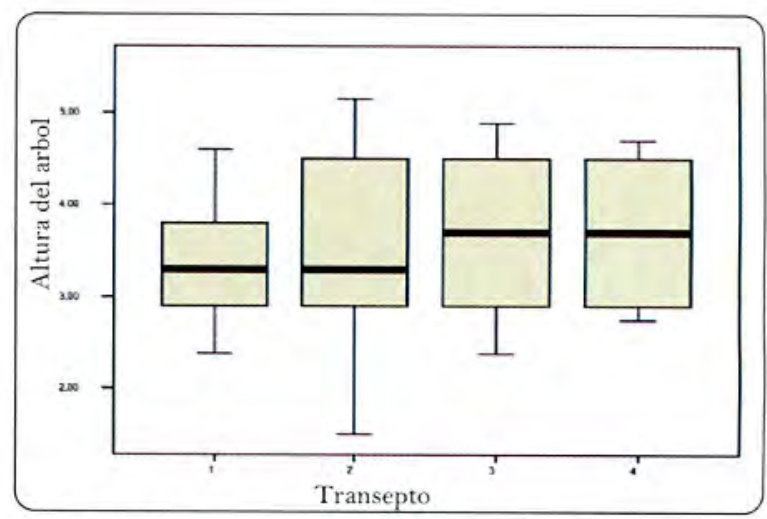

Gráfico $\mathrm{N}^{\circ}$ o2: Promedio de altura del árbol de polylepis rugulosa por transepto, provincia Candarave 2008-2009 en metros. 


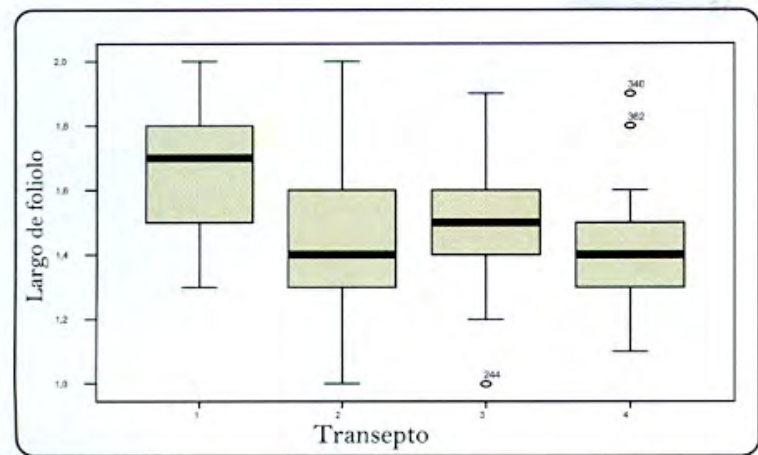

Gráfico $\mathrm{N}^{\mathbf{0}}$ os: Promedio del largo del foliolo de polylepis rugulosa por transepto, provincia Candarave 2008-2009 en centímetros.

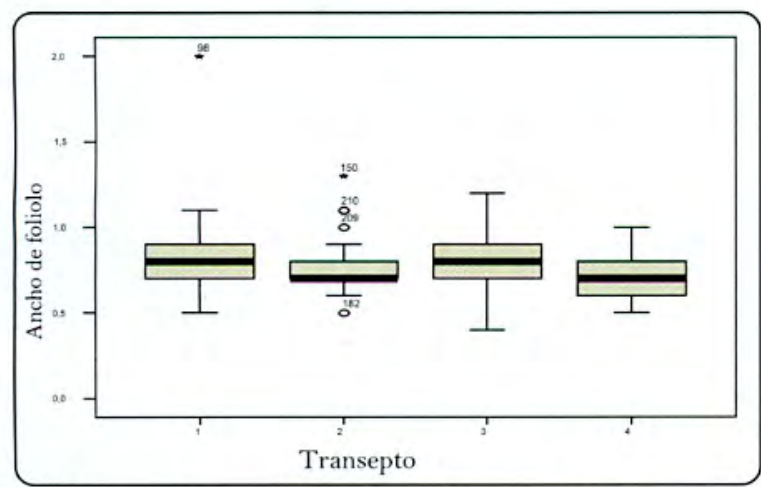

Gráfico $\mathbf{N}^{\circ}$ 04: Promedio del ancho del foliolo de polylepis rugulosa por transepto, provincia Candarave 2008-2009 en centímetros.

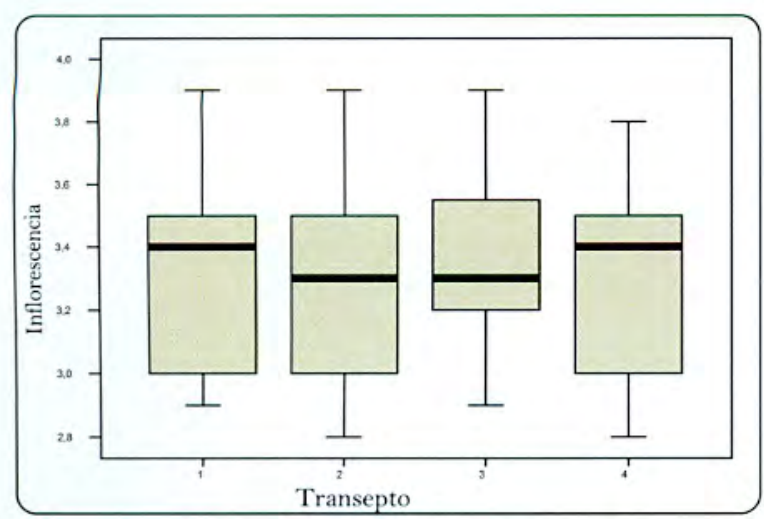

Gráfico $\mathrm{N}^{\mathbf{o}}$ 05: Promedio de la longitud de la inflorescencia de polylepis rugulosa por transepto provincia Candarave 2008-2009 en centímetros.

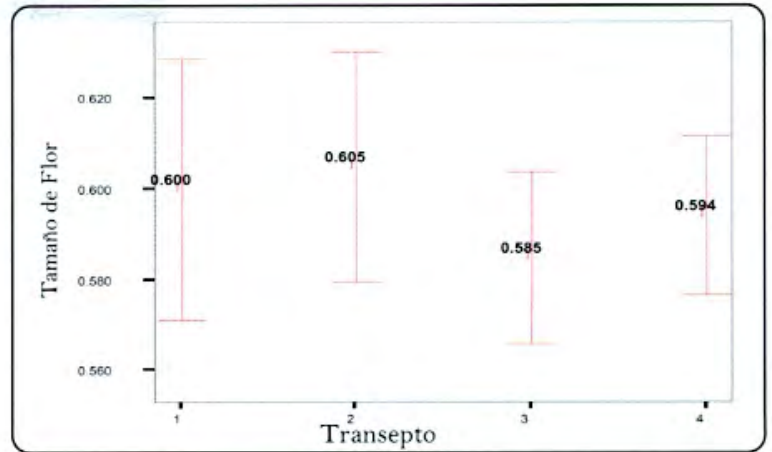

Gráfico $\mathbf{N}^{\mathbf{0}}$ o6: Promedio del tamaño de flor de polylepis rugulosa por transepto, provincia Candarave 2008-2009 en centímetros.

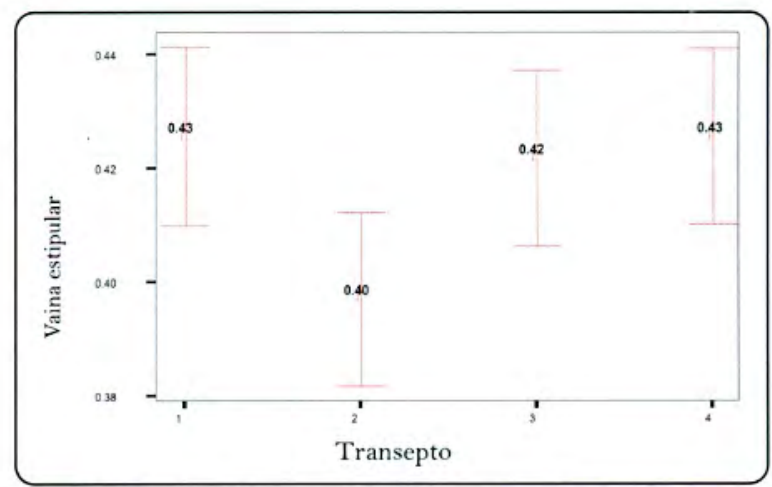

Gráfico $\mathbf{N}^{\mathbf{0}}$ 07: Promedio del tamaño de vaina estipular de Polylepis Rugulosa por transepto provincia Candarave 2008-2009 en centímetros.

Nuestro estudio evidenció resultados importantes en cuanto a las características fenotípicas de la Queñoa en la zona altoandina de la Provincia de Candarave, antes denominada Polylepis besseri. La aplicación de una nueva clave "Taxonomía y Distribución de Polylepis (Rosaceae)" de Michael Kessler, Alexander N. Schmidt-Lebuhn (2005) motivó el desarrollo de la presente investigación, y se logró medir y caracterizar la especie de Polylepis en la zona estudiada.

Por lo tanto, luego de los resultados de una serie de mediciones de características fenotípicas, tanto de variables cuantitativas y cualitativas, llegamos a concluir que la nominación de la especie 
Polylepis besseri no es la correcta para esta zona andina. Nuestro estudio, de manera fehaciente, lo identifica como Polylepis rugulosa, en razón de los siguientes resultados:

En lo que respecta a la altura de Polylepis rugulosa se observó una gran variabilidad y que en los transeptos muestreados, estos varían rotundamente y que se encontraron árboles desde $1,5 \mathrm{~m}$, el más pequeño y $5,2 \mathrm{~m}$. el de mayor tamaño. Cabe destacar los valores encontrados no distorsionan el criterio para incriminar dentro de lo establecido en la clave.

En la ficha de antecedentes descrita por Muñoz \& Serra (2006), ha sido caracterizado como un árbol o arbusto de $3-5 \mathrm{~m}$ de alto, con un promedio de $4 \mathrm{~m}$; en nuestro trabajo de investigación el promedio de los 4 transeptos fue de $3.53 \mathrm{~m}$. Este resultado es casi homogéneo en cada unidad de muestra. Por observaciones hechas, no hay árboles más altos para este bosque y que la característica fenotípica es propia de Polylepis rugulosa, pues coincide con los criterios establecidos en la clave; pero esta especie difiere de la especie Polylepis besseri, pues estudios hechos por Fjeldsa y Kessler (1996), Fjeldsa y Krabbe (1990), describen que la altura varia de 3 a $10 \mathrm{~m}$, con un promedio de $7 \mathrm{~m}$., es decir, superior al promedio dado para Polylepis rugulosa.

El foliolo es la característica más importante en la identificación de especies de Polylepis sp., ya que nos dan indicios de la especie para su identificación y estos varían de especie a especie. Kessler en la clave de identificación señala que $P$. rugulosa tiene 2 a 4 pares de foliolos. En nuestro trabajo se encontró 2 a 4 pares de foliolos en cada unidad de muestra; Simpson, J. da P. besseri halló 1 a 3 pares de foliolos, y en el largo del foliolo se observó una gran variabilidad. El foliolo varia en su forma. Unos son ovalados, obovados y circulares, según describe Kessler(1995), Kessler \& SchmidtLebuhn(2006). Sin embargo, los valores encontrados no distorsionan el criterio para incriminar dentro de lo establecido en la clave.

Estudios realizados por Kessler \& SchmidtLebuhn (2005) en la clave "Taxonomia y Distribucion de Polylepis (Rosaceae)" dan valores de 1,1-2,3 cm. del largo del foliolo para Polylepis rugulosa; el promedio encontrado en el presente trabajo es de $1,51 \mathrm{~cm}$. Este valor varía en cada uno de las unidades muestrales, teniendo como valores mínimos $1 \mathrm{~cm}$. y como máximo $2 \mathrm{~cm}$. El promedio está dentro del rango señalado por la clave. Así mismo, respecto al ancho del foliolo, la clave no muestra valores mínimos ni máximos. Pero podemos afirmar que los promedios resultantes en estos transeptos son casi homogéneos, pues el promedio del ancho del foliolo es $0,76 \mathrm{~cm}$; el rango mínimo, $0,4 \mathrm{~cm}$. y el máximo, $2 \mathrm{~cm}$.

En un estudio que realizó Muñoz \& Serra, (2006) dan valores del ancho de foliolo, siendo el rango 1,8-3,5 cm. Estos valores comparados con los datos obtenidos también están dentro del rango del ancho del foliolo. El dato de Polylepis rugulosa debe ser tomado en cuenta para futuros trabajos puesto que no se encuentran en la clave taxonomica de Kessler \& Schmidt-Lebuhn (2005).

Los resultados de las características cualitativas para esta especie son: foliolos trifoliados, imparipinnadas con superficie superior del foliolo fuertemente rugosa; pelos glandulares blancos, los folíolos con el envés densamente lanoso, foliolos ovalados, obovados a circulares, brillantes en el haz, con pelos blanquecinos en el envés; raquis lanoso. Estos resultados son descritos por Kessler \& Schmidt-Lebuhn (2005) en la clave "Taxonomia y Distribucion de Polylepis (Rosaceae)"para la especie Polylepis rugulosa. Por el contrario, la especie polylepis besseri tiene las siguientes características: Superficie superior del foliolo ligeramente a moderadamente rugosa; pelos glandulares amarillos, sobre el raquis de la hoja $\mathrm{y} / \mathrm{o}$ superficie inferior del foliolo. Como podemos ver, al aplicar los criterios de la clave, tanto $\mathrm{P}$. besseri y P. rugulosa son notablemente diferentes en suidentificación.

Los valores del tamaño de las vainas estipulares encontradas en el presente trabajo de investigación demostró un promedio de $0,42 \mathrm{~cm}$. teniendo como $0,3 \mathrm{~cm}$. y el máximo $0,6 \mathrm{~cm}$. Estos datos expuestos no se encuentran establecidos en la clave, pero concuerdan con las características $\mathrm{P}$. rugulosa. En cuanto a los atributos cualitativos sólo existe una ficha de antecedentes descrita por Muñoz \& Serra, (2006), donde expone que el ápice es protuberante $\mathrm{y}$ densamente panoso $\mathrm{o}$ lanoso, tal como se encontró en cada una de las unidades muestreadas. Estos resultados también excluyen a P. besseri ya que presentan vainas estipulares carentes de espolones o con espuelas débiles de hasta $1 \mathrm{~mm}$ de largo.

En cuanto a la inflorescencia, Kessler \& Schmidt-Lebuhn (2005) en la clave "Taxonomia y Distribucion de Polylepis (Rosaceae)" no hace constar el tamaño. En el presente trabajo evidenciamos como promedio $3,34 \mathrm{~cm}$. y damos como valor mínimo 2,8 y máximo $3,9 \mathrm{~cm}$. En esta característica cualitativa, la clave no dimensiona los valores cuantitativos, pero sí, la describe como inflorescencia prácticamente carentes de pelos glandulares amarillos cubiertos de pelos blancos, lo cual concuerda con los resultados observados en 
P. rugulosa. Estas características notablemente difieren de $\mathrm{P}$. besseri, pues tienen pelos glandulares amarillos presentes sobre las inflorescencias.

Otra de las características que la clave no da valor cuantitativo es el tamaño de la flor; sin embargo en nuestro trabajo de investigación presentamos un promedio de $0,59 \mathrm{~cm}$. teniendo como valor mínimo 0,5 y máximo $0,7 \mathrm{~cm}$., cubier to de pelos blancos que no se ven fácilmente. Los resultados son descritos por Kessler \& SchmidtLebuhn (2005) en la clave "Taxonomia y Distribucion de Polylepis (Rosaceae)" como la especie P. rugulosa y que varían con P. besseri, porque tienen pelos glandulares amarillos presentes en la flor.

Los frutos secos no se ven fácilmente, ya que es difícil observar en forma macroscópica. No se puede caracterizarlo, fenotípicamente y es necesario un estereoscopio para poder hacerlo.

Se observó que el fruto es de color naranja marrón, aquenio lanoso, con 4 proyecciones planas de forma irregular y varias puntas, cubiertas de pelos glandulares blancos, con varias espinas. Este resultado se comprueba con la clave de Kessler \& Schmidt-Lebuhn (2005) y también con los resultados del estudio de Muñoz \& Serra(2006). Ambos coinciden con los resultados obtenidos en la presente investigación, en cambio la especie de P. besseri presenta pelos glandulares amarillos en el fruto, siendo esta la principal diferencia entre ambas especies.

Las especies de Polylepis se distinguen por sus frutos, ya que presentan diferencias fenotípicas.

Este es el caso de P. subtusalbida, pues el fruto tiene crestas finamente dentadas verde o marrón, mientras que P. tarapacana tiene de 3 a 5 crestas duras irregulares y que P. subsericans fue el fruto con crestas aplanadas irregularmente, tal como precisa Kessler \& Schmidt-Lebuhn (2005). Estas características fenotípicas comparadas con la especie P. rugulosa difieren notablemente unas de otras.

\section{Análisis de características fenotípicas cualitativas}

En lo que respecta a los resultados de las características cualitativas de la especie P. rugulosa para la zona altoandiana de Candarave, se observaron los siguientes rasgos morfológicas: árboles con troncos torcidos, corteza delgada y exfoliante, de color marrón rojizo que se desprende en pedazos largos.
Los foliolos son trifoliados, imparipinnadas con superficie superior fuertemente rugosa; pelos glandulares blancos, con el envés densamente lanoso, foliolos ovalados, obovados a circulares, verde brillante en el haz, con pelos blanquecinos en el envés; raquis lanoso.

Respecto a las características de las vainas estipulares, estas presentan un ápice protuberante y densamente lanoso en toda su morfología. La inflorescencia y la flor están cubiertas de pelos blancos. La flor es de color rojo cereza. Es dialipétala con 3 a 4 pétalos ligeramente ovados a elípticos, flor bisexual. El hipantio está formado de las partes básicas sépalos, pétalos y estambre. Los pétalos son igual en número que los sépalos. Los frutos son secos. No se ven fácilmente. Un fruto aquenio lanoso, tiene 4 proyecciones planas de forma irregular con espinas de color negro $y$ cubiertos de pelos blancos.

\section{CONCLUSIONES}

- La especie en el bosque nativo de la provincia de Candarave -Tacna presenta las siguientes características: el árbol muestra una altura de $3,53 \mathrm{~m}$. de promedio, troncos torcidos, corteza delgada y exfoliante, de un color marrón rojizo, que se desprenden en pedazos largos.

- Los foliolos tienen un promedio de $1,51 \mathrm{~cm}$. de largo y $0,76 \mathrm{~cm}$. de ancho. Son trifoliados, imparipinnadas con superficie superior del foliolo fuertemente rugosa; pelos glandulares blancos, con el envés del foliolo densamente lanoso, foliolos ovalados, obovados a circulares, verde brillantes en el haz, con pelos glandulares blanquecinos; y raquis lanoso.

- Las vainas estipulares tienen un tamaño de $0,42 \mathrm{~cm}$. de promedio. Presentan un ápice protuberante y que es densamente lanoso en toda su morfología; resultados que comparados con la clave de Kessler \& Schmidt-Lebuhn (2005) coinciden con la especie Poylepis rugulosa.

\section{REFERENCIAS BIBLIOGRÁFICAS}

Argollo, J. y otros. (2004). Potencialidad Dendrocronológica de Polylepis Tarapacana en los Andes Centrales de Bolivia”, Mendoza, Argentina.

Armesto, J. y otros (1994). Ecología de los bosques nativos de Chile. Editorial Universitaria. Santiago, Chile. 
Cáceres, C. (2004). Taxonomia y Usos Tradicionales de las Plantas Medicinales de la Provincia de Candarave. Tacna - Perú. Tesis para optar el título profesional de Biólogo Microbiólogo, Escuela de Biología Microbiología, Facultad de Ciencias de la Universidad Nacional Jorge Basadre Grohmann. Tacna.

Calderón, G. (1993). Reproducción de la Queñoa (Polylepis besseri). Un reto superado. Chile Forestal. Ficha de antecedentes, chile.

Charaja, F. (2004). Investigación científica. Puno-Perú.

Franco, J. (2003). Importancia del Bosque de Queñoas para el Desarrollo Sostenible de las Comunidades de la Provincia de Candarave", Tacna - Perú. (Tesis para optar el grado de Magister. Escuela de Post grado de la Universidad Nacional Jorge Basadre Grohmann, Tacna.

Kessler, M. (1995a). Revalidación de Polylepis rugulosa Bitter (Rosaceae). Revista científica. Gayana Bot.

Kessler, M. (1995b). The genus Polylepis (Rosaceae) in Bolivia. Revist Cientifica. Candollea.

Kessler, M. (2006). Bosque de Polylepis. Universidad Mayor San Andrés. La PazBolivia. resumen.

Kessler, M. y Schmidt-Lebuhn, A. (2005). Taxonomia y Distribución de Polylepis ( $\mathrm{R}$ o s a c e s ), A l e m a n i a . http://www.senckenberg.de/odes/0601.htm. Información proporcionada por el autor.

Kowoll, M. (1993). Antecedentes y caracterización de las poblaciones de queñoa Polylepis besseri Hieron entre Chapiquiña y Tignamar en la precordillera de la I región. Santiago-Chile.

Ministerio de Agricultura, Instituto Nacional de Recursos Naturales (2004). Monitoreo de la Biodiversidad. Lima-Perú.

URL:http://cdc.lamolina.edu.pe/Descargas/ anp/bib_gp_monitoreo.html

Lemus, J. (2006). Fenología Reproductiva en Tres Tipos de Vegetación de la Planicie Costera de la Península de Paraguana, Edit. Asociación Venezolana para el Avance de la Ciencia Venezuela. Caracas - Venezuela.
Patterson, W. (2004). Polylepis besseri Hieron. Part. II Species Descriptions.

Renison, I. y otros. (2004). Anthropogenic soil degradation affects seed viability in Polylepis australis mountain forest of central Argentina. Forest Ecologya and Mangement

Rodríguez, R, y otros (1989). Flora Arbórea de Chile. Universidad de Concepción. Editorial de la Universidad de Concepción. Santiago. Chile.

Simpson, B. (1979). A Revision Of The Genus Polylepis (rosacea: sanguisorbeaceae). Washington, Smithsonian Institution press.

Smith, A. (1977). Establishment Of Seedlings of Polylepis Sericea In The Paramo (Alpino) Zone Of The Venezuela Andes.

Simpson, B. (1986). Speciation and specialization of Polylepis in the Andes. In: Vuilleumier, F., Monasterio, M. (Eds.), High Altitude Tropical Biogeography. Oxford University Press, Oxford.

Túlume, M y otros. (2004). Reporte. Caracterización del Bosque Nativo de Queñoas (Polylepis Besseri) en las Comunidades de Muylaque - Sijuaya, San Cristóbal, Moquegua-Perú.

Valla, J. (1999). Morfología de las Plantas Superiores. Edit. Hemisferio Sur, Buenos Aires, Argentina.

Velásquez, A. (1988). Evaluación Forestal de Los Bosques Naturales del Genero Polylepis (Queñoas o Lampayas) de la Provincia de Lampa, Departamento de Puno. Universidad Nacional San Antonio Abad del Cusco, Perú.

Yallico, E. (1992). Distribución de Polylepis en el Sur de Puno, Proyecto Árbol Andino, Puno-Perú.

\section{Correspondencia:}

Luis Morales Aranibar

Ciudad Universitaria fundo "Los Granados"

Av. Miraflores s/n Tacna - Perú

Giovanni Aragón Alvarado

Ciudad Universitaria fundo "Los Granados"

Av. Miraflores s/n Tacna - Perú

Edith Silva Flores

Ciudad Universitaria Fundo "Los Granados" Av. Miraflores s/n Tacna-Perú 
ANEXOS: IMÁGENES EN RELACIÓN AL ARTÍCULO CIENTÍFICO
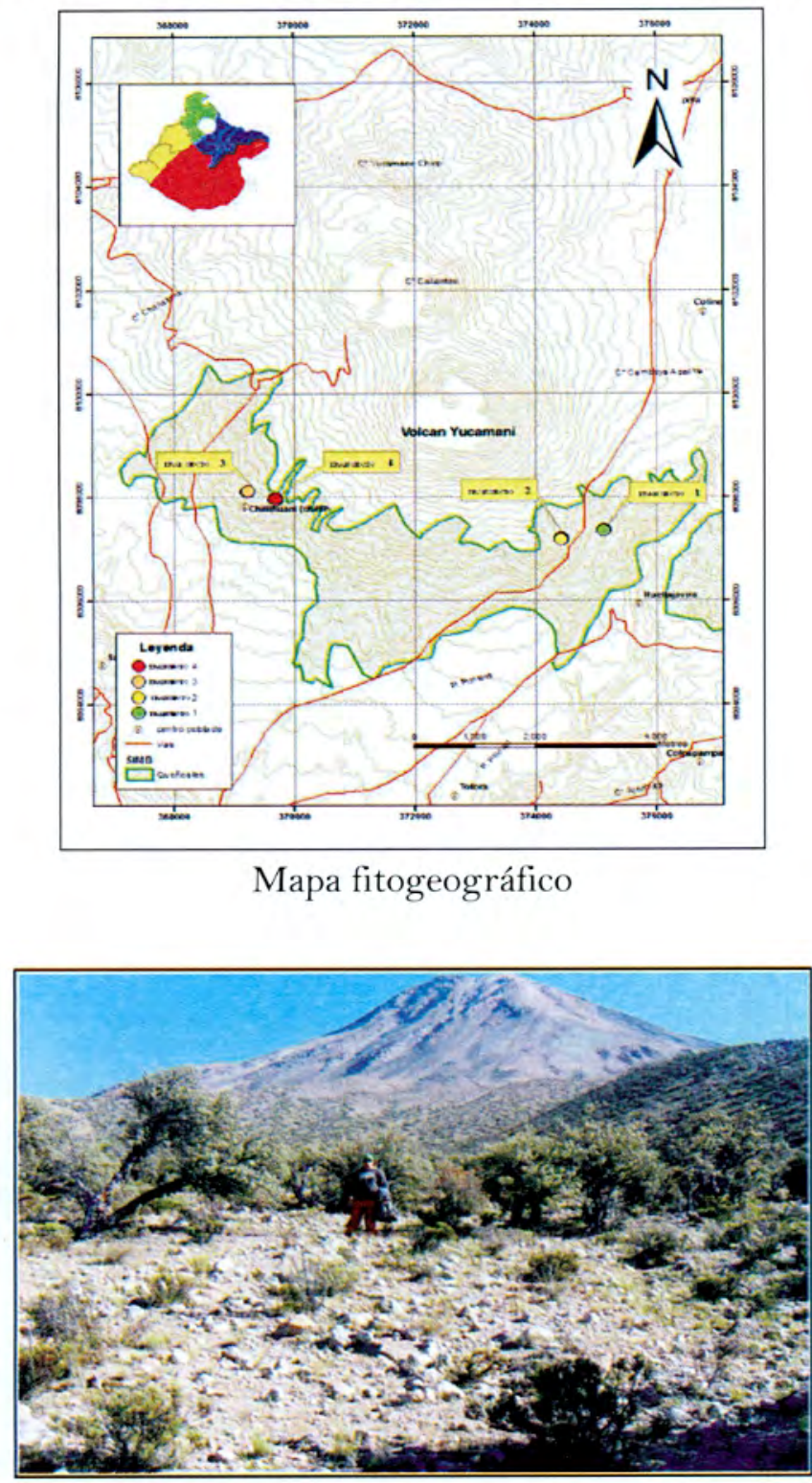

Volcán Yucamani

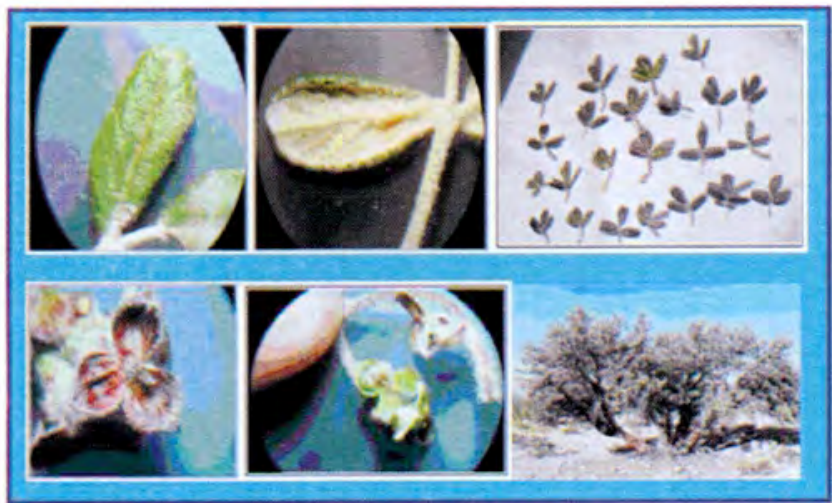

Caracterizaciones fenotípicas 